\title{
Antibiotic-Induced or Aspergillus Infection Related Acute
} Interstitial Nephritis?

\begin{tabular}{|l|l|l|}
\hline E. & Edith & Renoult $^{\mathrm{a}}$ \\
\hline B. & Bernadette & Aymard $^{\mathrm{b}}$ \\
\hline F. & François & Chabot $^{\mathrm{c}}$ \\
\hline D. & Dominique & Hestin $^{\mathrm{a}}$ \\
\hline N. & Nicolas & Delorme $^{\mathrm{c}}$ \\
\hline L. & Lyliane & Kures $^{\mathrm{d}}$ \\
\hline J.-M. & Jean-Marie & Polu $^{\mathrm{c}}$ \\
\hline M. & Michèle & Kessler $^{\mathrm{a}}$ \\
\hline
\end{tabular}

aService de Néphrologie, 'Laboratoire d'Anatomie et de Cytologie, Pathologiques, ${ }^{\mathrm{c}}$ Service des Maladies Respiratoires et de Réanimation Respiratoire, et ${ }^{\text {d }}$ Laboratoire Central de Parasitologie et Mycologie, Centre Hospitalo-Universitaire de Nancy, France

Dr. Edith Renoult, Service de Néphrologie, Centre Hospitalier Universitaire de Brabois, F-54511 Vandœuvre-lès-Nancy (France)

Dear Sir,

Renal impairment in aspergillosis is the result of distinct processes: (1) isolated Aspergillus cast of the renal pelvis; (2) a pan-urothelial ascending infection, or (3) disseminated hematogenous spread. This latter form could be suspected in a patient who developed acute renal failure with granulo-matous interstitial nephritis and who died with a disseminated aspergillosis and a Pseudomonas aeruginosa superinfection.

A 60 -year-old man who had a history of chronic obstructive pulmonary disease was admitted because of acute renal failure. The patient was first admitted to his local hospital because of acute respiratory failure secondary to a bronchopulmonary infection. His condition required mechanical ventilation, antibiotics (cefotaxime and ofloxacin), and high-dose corticosteroids for 15 days. On day 21 , the patient was dismissed from the hospital without any antibiotic or steroid therapy. Two days later, he was admitted to our center suffering from diarrhea, abdominal pain, macroscopic hematuria, and decreased urine output. He was afebrile. The physical examination showed nothing abnormal except for generalized edema. Although the blood chemistry was initially normal, laboratory data showed urea nitrogen $45.6 \mathrm{mmol} / 1$, sodium 109 , potassium 4.1 , chloride 72, bicarbonate $22 \mathrm{mmol} / 1$, creati-nine $700 \mu \mathrm{mol} / 1$, protein $48 \mathrm{~g} / \mathrm{l}$, and uric acid 460 $\mathrm{mol} / \mathrm{l}$. Proteinuria was $0.50 \mathrm{~g} / \mathrm{day}$. The hemoglobin concentration was $12.1 \mathrm{~g} / 100 \mathrm{ml}$, the white blood cell count $20.1 \times 109 / 1$ (97.6\% neutrophils, $0 \%$ eosinophils), and the platelet count was $289,000 / \mathrm{mm} 3$. The serum IgE level was elevated (333 kUI/1; normal range $0-150)$. The results of the following examinations were normal or negative: antinuclear antibodies, C3, C4, total hemolytic complement, and circulating immune complexes. Renal ultrasonography revealed no abnormalities.

Renal biopsy performed 3 weeks after the end of antibiotic and steroid therapy showed acute interstitial nephritis with interstitial infiltration of mononuclear cells. Three focal 
tubulointerstitial lesions were notable: they appeared as small foci of plasmocytes, histio-cytes, small lymphocytes with degeneration or necrosis of unidentified (tubular?) cells, and large amounts of an eosinophilic amorphous substance (fig. 1). Special stains were negative for amyloidosis and for microorganisms, especially mycobacteria and fungi. Glo-merular and vascular changes were not noticeable by light microscopy. The renal tissue was examined using direct immunofluores-cence staining and rabbit anti-IgM C3-Clq, fibrinogen serum, goat antiIgA serum, and rabbit anti-IgG and albumin sera. Immuno-globulin or complement depositions were not found. This morphologic appearance was considered suggestive of an antibioticinduced or infectious disease. Serological tests for Toxoplasma, Candida, Brucella, Legio-nella, Mycoplasma, Leptospira, Chlamydia, and Aspergillus spp. and for Epstein-Barr virus, cytomegalovirus, adenovirus, coxsackie-virus, parainfluenza viruses, hepatitis A and B viruses, and Hantaan and human immunodeficiency viruses were negative.

Chest radiography showed an irregular nodular infiltrate in the right upper lobe. Fiberoptic bronchoscopy showed an epiglot-tic excrescence and inflammatory endobron-chial changes without any tumor. Histologi-cal examination of a transbronchial lung biopsy specimen from the right upper lobe revealed interstitial fibrosis and hemosider-in-laden macrophages.

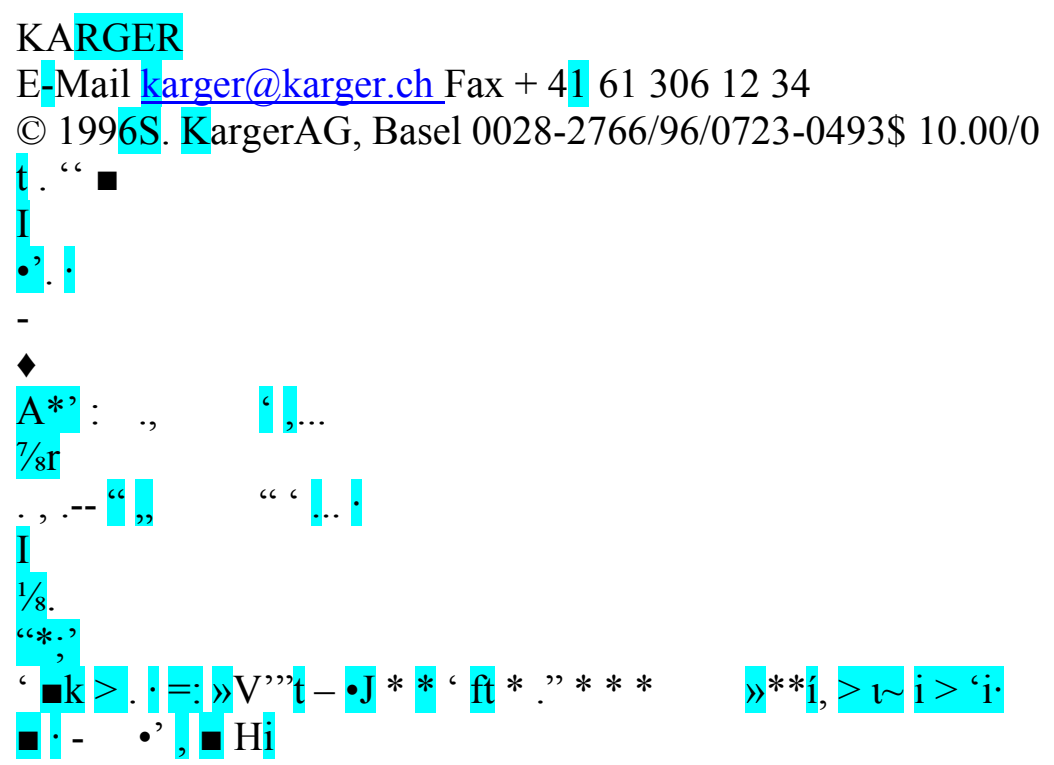

Fig. 2. A few fragmented branching septate hyphae (arrows) sug-

Fig. 1. Renal granulomatous lesion with epithelioid cells and mul-

tinucleated giant cells surrounding a central necrotic area (asterisk). gest'mg Aspergillusv/ere present in renal granulomatous lesions. PAS.HE-saffron. $\times 80 . \quad \times 500$.

Renal failure did not improve and required hemodialysis. A second renal biopsy was performed. The microscopy results were similar to those of the first biopsy specimen. Seven days after admission, the patient developed acute tension pneumothorax. He became febrile, and the bronchitis exacerbated. Blood and urine cultures for bacterial pathogens were negative. Culture of sputum yielded $\mathrm{P}$. aeruginosa. The patient was treated intravenously with pefloxacin and amikacin, with no evident effect. A computed tomography scan showed bilateral perinephric masses. An aspirate of the right collection revealed purulent fluid. Cultures grew P. aeruginosa. Percutaneous drainages of the abscesses were made. The patient's clinical condition steadily deteriorated, and he died 43 days after admission. Autopsy revealed disseminated aspergillosis 
with a small peribronchial abscess in the apex of the right lung and papillary necrosis and numerous microabscesses in both kidneys. Histo-logically, some of the abscesses had a tuberculoid appearance with epithelioid cells and multinucleated giant cells surrounding a central necrotic area. A few fragmented branching septate hyphae suggesting Aspergillus were detected within the necrotic area after periodic acid-Schiff and silver staining (fig. 2). There was no evidence of pelvic caly-ceal obstruction by bezoar. Postmortem cultures for fungi were not performed.

In reviews of granulomatous interstitial nephritis [1], the most common associated conditions are drug ingestion, infections,

sarcoidosis, and vasculitis. To our knowledge, aspergillosis is rarely mentioned. In this case, the diagnosis of drug-induced acute interstitial nephritis could be suggested: renal failure associated with hematuria occurred after treatment with ciprofloxacin and cefotaxime which have both been reported to cause acute interstitial nephritis [1]. Renal biopsy showed a diffuse infiltration of the interstitium. However, the bron-chopulmonary abnormalities and the early pathologic renal findings such as the foci of cortical necrosis (uncommon in drug-related interstitial nephritis), all could initially suggest an infectious process. Diagnosis of disseminated aspergillosis with pulmonary and renal involvement was made at autopsy by demonstrating fungal elements in the tissues [2]. Unfortunately, the hyphae of the Aspergillus species were absent in the two antemortem renal biopsy specimens. Classically, invasive aspergillosis occurs in severely immunosuppressed patients [2]. Recent high-dose corticoid treatment and broad-spectrum antimicrobial therapy could have predisposed the patient to invasive disease. Disseminated aspergillosis usually causes an acute pyogenic reaction combined with necrosis [3].

Granulomatous lesions are rare in immunocompromised hosts, but have been noted in apparently healthy patients with disseminated aspergillosis $[2,4]$ and were observed in this patient. In this case, Aspergillus played an important but not exclusive role in the fatal outcome. Concomitant $P$. aeruginosa nosoco-

mial infection was deleterious. Severe Candida or Pseudomonas infections are often associated with aspergillosis [2] and worsen the unfavorable prognosis of invasive aspergillosis.

In conclusion, in view of the complex drug and infection history in our patient, ascertainment of the etiologic part of Aspergillus in acute interstitial nephritis is difficult. However, the possibility of disseminated aspergillosis should be considered when evaluating patients with acute granulomatous interstitial nephritis of unclear etiology, particularly when there is an associated lung involvement or risk factors (immunodeficiency, corticotherapy). The outcome of this devastating opportunistic fungal disease is directly dependent on early diagnosis and therapy. References

Bennett WN, Elzinga LW, Porter GA: Tubu-lointerstitial and toxic nephropathy; in Brenner BM, Rector FC (eds): The Kidney. Philadelphia, Saunders, 1991, pp 1430-1496. Rinaldi MG: Invasive aspergillosis. Rev Infect Dis 1983;5:1061-1077.

Bibler MR, Gianis JT: Acute ureteral colic from an obstructing renal aspergilloma. Rev Infect Dis 1987;9:790-794. Hillerdal G, Benson L, Lindgren A, Hjertquist SO: Disseminated pulmonary aspergillosis in a previously healthy young man. Scand J Infect Dis 1984;16:217-222.

494

Nephron 1996;72:493-494

Renoult/Aymard/Chabot/Hestin/Delorme/ Kures/Polu/Kessler 